\title{
A new approach to lymphedema following breast cancer treatment with lymphatic endothelial cell markers
}

\author{
(D) Nilhan Nurlu ${ }^{1}$, GGül Tuğba Bulut ${ }^{2}$ \\ ${ }^{1}$ Gaziosmanpaşa Taksim Training and Research Hospital, Department of Clinical Biochemistry, İstanbul, Turkey \\ ${ }^{2}$ Gaziosmanpaşa Taksim Training and Research Hospital, Department of Physical Medicine and Rehabilitation, İstanbul, Turkey
}

Cite this article as: Nurlu N, Bulut GT. A new approach to lymphedema following breast cancer treatment with lymphatic endothelial cell markers. J Health Sci Med 2021; 4(3): 322-328.

\begin{abstract}
Aim: Lymphedema (LE) is a common iatrogenic complication of breast cancer treatment that may occur following axillary lymph node dissection and radiotherapy. In this study, we aimed to investigate the serum levels of the lymphatic endothelial cell spesific (LECs) markers; homeobox transcription factor (Prox-1), lymphatic vessel endothelial receptor-1 (LVYE-1), and podoplanin (PDPN) in patients with LE following breast cancer treatment.

Material and Method: In total, 44 female patients who developed LE in an upper extremity after breast cancer treatment constituted the study's LE group, and 44 healthy women constituted the control group. Patients' arm circumferences were measured, and the differences between the sums of arm circumferences (DSOAC) were accepted as indicating LE when the difference was $2 \mathrm{~cm}$ or $10 \%$. Serum Prox-1, LVYE-1,PDPN concentrations were measured using commercial enzyme-linked immunosorbent assay (ELISA).

Results: Prox-1, LVYE-1, and PDPN levels were significantly lower in the LE group than in the control group ( $\mathrm{p}<0.01, \mathrm{p}=0.02$, $\mathrm{p}=0.04$, respectively). Prox- 1 levels were found to be significantly higher in patients with Stage $1 \mathrm{LE}$ than those with Stage $2(\mathrm{p}<0.001)$. There was a weak negative correlation between Prox-1 levels and DSOAC and number of axillar lymph node removed (NALNR) levels ( $r=-0.417$ and -0.426 , respectively; $\mathrm{p}<0.01$ ), and a moderate positive correlation between DSOAC and NALNR $(\mathrm{r}=0.533, \mathrm{p}<0.001)$.

Conclusion: Prox-1, LVYE-1, and PDPN levels decrease in secondary LE. Further investigations of LEC markers may aid the development of new perspectives on LE diagnosis, prognosis, and treatment.
\end{abstract}

Keywords: Lymphatic endothelial cell markers, secondary lymphedema, lymphangiogenesis

\section{INTRODUCTION}

Lymphedema (LE) is the accumulation of proteinrich fluid in the interstitial compartment followed by inflammation, adipose tissue hypertrophy, and fibrosis. LE occurs as a result of impaired lymphatic drainage, and is a chronic and progressive disease that causes psychological, social, and economic problems, increasing patient discomfort. Patients with LE complain of pain, weight sensation, progressive swelling of the affected limb, decreased limb function, and a generally reduced quality of life (1-3).

In developed countries, secondary LE is now more common than primary LE is because effective cancer treatments have increased patient survival rates. In the United States, LE affects one patient out of every six treated for solid tumors. Furthermore, with increasingly effective oncologic therapies, the prevalence of LE is expected to increase as patient survival increases $(2,4)$. More recently, attention has been drawn to the fact that most women with breast cancer suffer from upper-extremity LE, especially after surgery and radiation therapy (5-7).

LE is diagnosed via anamnesis and physical examination. Non-invasive methods, such as bioelectric impedance analysis, may also be used for diagnosis during physical examination. However, there is currently no specific marker used for diagnosis. After manual lymphatic drainage and multi-layered bandage sessions, conservative treatment with compression garments stops the progression of the disease, although there is currently no definitive treatment for $\operatorname{LE}(1,8)$. The absence of more effective curative treatments and predictors of LE development is due to poor understanding of the underlying molecular mechanisms of LE. Further research to elucidate the pathophysiology of the disease may allow the development of new approaches for the diagnosis, prognosis, and treatment of the disease. 
Lymphatic capillaries are composed of lymphatic endothelial cells (LECs), which are connected to the basement membrane via anchor filaments, and are responsible for maintaining vascular patency (9). The lymphatic vascular system plays an important role in maintaining the interstitial fluid balance, in the trafficking of immune cells and immune surveillance, and the absorption of dietary fats through intestinal lymphatics. Most of the studies conducted to elucidate the pathophysiology of LE have focused on LECs. Although the lymphatic system was identified centuries ago, significant progress in our understanding of the mechanisms controlling the lymphatic system has been made in recent years following the discovery of LEC markers, gene analysis, and growth factors. The first identified LEC markers are prospero-related homeobox gene-1 (Prox-1), lymphatic vessel endothelial receptor-1 (LVYE-1), podoplanin (PDPN), and vascular endothelial growth factor receptor-3 (VEGFR-3) $(10,11)$.

LECs can adapt their shape and transport mechanisms to provide fluid removal in situations such as increased lumen pressure and shear stress $(12,13)$. LEC proliferation is induced via the application of increased intraluminal load during LE pathogenesis, and impaired lymphangiogenesis is subsequently observed. Insufficient lymphatic drainage and increased interstitial pressure gradually make lymphatic endothelial cells (LECs) dysfunctional, as these further impair their absorption and fluid handling capabilities. After a period of time, LE worsens because of lipid accumulation, lymphatic immobilization, and reduced trafficking of immune cells $(14,15)$. VEGFR-3 and LVYE-1 are known to play a role in physiological and pathological lymphangiogenesis $(16,17)$. Prox-1 is an executive gene that controls lymphatic cell-specific differentiation and provides transcriptional regulation of PDPN expression in LECs. PDPN is strongly expressed in the lymphatic endothelium, and the development of LE has been reported in PDPN null mice $(10,18)$.

Despite previous studies examining factors associated with LE severity and cytokine candidate genes that predict LE following breast cancer treatment $(19,20)$, to the best of our knowledge, no study of LEC markers has yet been conducted. The aim of the present study is to investigate the roles of Prox-1, LVYE-1, and PDPN in LE following breast cancer treatment.

\section{MATERIAL AND METHOD}

\section{Study Participants}

This study was carried out in accordance with the principles of the Declaration of Helsinki, as well as sound clinical training suggestions. Clear and full written consent was obtained from each patient with secondary LE following breast cancer treatment and each control subject, and the study was approved by the Gaziosmanpaşa Taksim Training and Research Hospital Clinical Researchs Ethics Committee (Date: 2018, Decision No: 81).

Forty-four female patients complaining of swelling in the ipsilateral arm after breast cancer treatment who were referred to Gaziosmanpaşa Taksim Training and Research Hospital, Lymphedema Polyclinic between January and July 2018 were included in the study. Cases of LE were diagnosed by anamnesis, physical examination, and measurements of the circumference of both arms of the patients (affected and unaffected). The medical records of the patients were consulted, and their self-evaluation reports were obtained through special interviews. All the patients underwent a total mastectomy, axillary lymph node dissection, and received both chemotherapy and radiotherapy. Excluded from the study were patients with active breast cancer or recurrence thereof, active upperextremity infections, lymphangitis, and ongoing cancer treatment. Further, 44 healthy, similarly-aged female volunteers from the staff of the same hospital who had no instance of systemic disease or LE were included as the control group for comparison in our investigation.

\section{The Differences Between the Sums of Arm Circumferences (DSOAC)}

The truncated cone method was used to measure the circumference along the affected (ipsilateral) and unaffected (contralateral) arm. Circumferences were measured at the 1st and 5th metacarpal, wrist (using the distal edge of the styloid process of the ulna and radius) and then every $5 \mathrm{~cm}$ along the arm. The sum of these circumferences was calculated, and the difference between the affected and unaffected arm was evaluated using the following formula:

$$
\operatorname{DSOAC}(\mathrm{cm})=\sum\left(I E_{c m p}-C E_{c m p}\right)
$$

$I E_{c m p}:$ Ipsilateral extremity circumference at the marked points

$\boldsymbol{C E}_{c m p}$ : Contralateral extremity circumference at the marked points

When the difference between the ipsilateral and the contralateral sides was more than $2 \mathrm{~cm}$, or more than $10 \%$, it was accepted as LE (21-23).

\section{LE Staging According to the International Society of} Lymphology (ISL) Staging System

This staging system is based on the clinical features of the disease, and classifies patients according to the presence or absence of limb swelling and pitting edema.

Patients with lymphatic damage and LE, but with no 
measurable limb volume/circumference changes, were classified as Stage 0 (latent LE); patients with measurable limb swelling and pitting edema improved by compression were classified as Stage 1 (Spontaneously reversible); patients with extreme swelling that cannot be relieved by compression because of fibroadipose accumulation were classified as Stage 2 (Not spontaneously reversible); patients with severe swelling and skin changes and endstage disease were classified as Stage 3 (24).

\section{Blood Sampling}

Venous blood samples taken from the LE and control groups were collected in the early morning after a minimum of 8 hours of overnight fasting. Then, the trial samples were centrifuged at $3000 \mathrm{x}$ g for 10 minutes, and the sera were preserved at $-40^{\circ} \mathrm{C}$ until just before analysis.

\section{Podoplanin, Prox-1, and LVYE-1 Measurements}

Serum Prox-1, LVYE-1 (SunRed Biological Technology Co. Ltd, Shanghai, China), and PDPN (Elabscience Biotechnology, Texas, USA) concentrations were measured using commercial enzyme-linked immunosorbent assays (ELISA).

\section{Statistical Analysis}

The Statistical Package for Social Sciences for Windows version 21 (IBM SPSS Inc, Chicago, Illinois) was used for the statistical analysis of the data. The KolmogorovSmirnov test was used to perform the normality control of the variables. Normally distributed mathematical variables were presented as means (SDs), whereas those not normally distributed were presented as medians (range).

To obtain detailed statistics, the mean, SD, median, minimum, maximum, frequency, and rates were used. To assess quantitative data, the Mann-Whitney $U$ test and independent sample t-test were applied. The Spearman's correlation test was used to examine the relationship between two variables. The $\chi 2$ test was used to assess qualitative data, in which a $\mathrm{p}$ value $<0.05$ was considered significant. A receiver operating characteristic (ROC) curve analysis was used to analyze the ability to predict the presence of LE based on the Prox-1, LVYE-1, and PDPN levels. Sensitivity and specificity values were determined when significant cutoff values were observed. A 5\% type 1 error rate was significantly predictive of the test variables when evaluating the area under the curve.

\section{RESULTS}

The mean age of the subjects with LE and those of the control group were not significantly different. No differences in cardiovascular risk factors such as hyperlipidemia, hypertension, diabetes mellitus, and smoking were detected between the groups $(p>0.05)$. The LE group's body mass index was significantly higher than that of the control group ( $\mathrm{p}=0.001$ ) (Table 1$)$. In the LE group,
Prox-1, LVYE-1, and PDPN levels were significantly lower than those of the control group $(\mathrm{p}<0.01, \mathrm{p}=0.02, \mathrm{p}=0.04$, respectively) (Table 2 ).

According to ISL [24], Prox-1 levels were significantly

\begin{tabular}{|c|c|c|c|}
\hline Characteristic & $\begin{array}{c}\text { Control } \\
\text { group } \\
(\mathrm{n}=44)\end{array}$ & $\begin{array}{l}\text { LE group } \\
(\mathrm{n}=44)\end{array}$ & $\mathbf{p}$ \\
\hline $\begin{array}{l}\text { Age (year) Median (Min- } \\
\text { Max) }\end{array}$ & $44(35-65)$ & $48(34-68)$ & $0.080^{*}$ \\
\hline $\begin{array}{l}\text { BMI }\left(\mathrm{kg} / \mathrm{cm}^{2}\right) \text { Median } \\
\text { (Min-Max) }\end{array}$ & $\begin{array}{l}24(21.5- \\
25.5)\end{array}$ & $28(22-45)$ & $0.001^{*}$ \\
\hline Diabetes mellitus & $4(9.1 \%)$ & $5(11.4 \%)$ & $0.637^{\star *}$ \\
\hline Hypertension & $2(4.5 \%)$ & $3(6.8 \%)$ & $0.540^{* *}$ \\
\hline Hypercholesterolemia & $1(2.3 \%)$ & $3(6.8 \%)$ & $0.080^{* *}$ \\
\hline Smoker & $4(9.1 \%)$ & $5(11.4 \%)$ & $0.733^{* *}$ \\
\hline
\end{tabular}

Table 2. Comparison of lymphatic endothelial cell marker levels of participants according to groups

\begin{tabular}{|lccccc|} 
& $\begin{array}{c}\text { Control Group } \\
(\mathbf{n = 4 4})\end{array}$ & $\begin{array}{c}\text { LE Group } \\
(\mathbf{n = 4 4 )}\end{array}$ & z & p \\
\cline { 2 - 3 } & $\begin{array}{c}\text { Median } \\
(\text { Min-Max })\end{array}$ & $\begin{array}{c}\text { Median } \\
(\text { Min-Max })\end{array}$ & & \\
\hline Prox-1 $(\mathrm{pg} / \mathrm{ml})$ & $556(396-752)$ & $455(335-663)$ & -4.1 & $<0.01$ \\
LYVE-1 $(\mathrm{ng} / \mathrm{mL})$ & $6.0(2.3-20)$ & $5.4(1.8-8)$ & -2.4 & 0.02 \\
PDPN $(\mathrm{ng} / \mathrm{mL})$ & $1.9(0.02-6.40)$ & $0.9(0.06-4.43)$ & -2.1 & 0.04 \\
\hline${ }^{*}$ Mann Whitney U test, Min: Minimum, Max: Maximum & & \\
\hline
\end{tabular}

higher in Stage $1 \mathrm{LE}$, and DSOAC and number of lymph nodes removed (NLNR) were significantly higher in Stage 2 LE $(\mathrm{p}<0.001)$. There were no significant differences in LVYE-1 and PDPN levels between LE stages 1 and 2 (Table 3). According to the Spearman correlation analysis, there was a weak negative correlation between Prox-1 levels and DSOAC and NLNR levels $(r=-0.417$ and -0.426 , respectively; $\mathrm{p}<0.01$ ) (Figures 1 and 2), whereas a positive and moderate correlation was seen between DSOAC and NLNR ( $\mathrm{r}=0.533, \mathrm{p}<0.001)$ (Figure 3).

\begin{tabular}{|c|c|c|c|c|}
\hline & \multicolumn{2}{|c|}{ ISL Stage } & \multirow{3}{*}{$\mathbf{Z}$} & \multirow{3}{*}{$\mathbf{p}$} \\
\hline & Stage $1(n=12)$ & Stage $2(n=32)$ & & \\
\hline & $\begin{array}{c}\text { Median } \\
\text { (Min-Max) }\end{array}$ & $\begin{array}{c}\text { Median } \\
\text { (Min-Max) }\end{array}$ & & \\
\hline $\operatorname{DSOAC}(\mathrm{cm})$ & $11.7(6-26)$ & $26.5(12-57)$ & -3.6 & $<0.001$ \\
\hline NLNR & $11(9-15)$ & $17(9-45)$ & -3.5 & $<0.001$ \\
\hline Prox-1 (pg/ml) & $598(533-663)$ & $433(336-508)$ & -5.1 & $<0.001$ \\
\hline LYVE-1 (ng/mL) & $5.4(1.8-8.0)$ & $5.4(2.0-7.4)$ & -0.1 & 0.93 \\
\hline PDPN (ng/mL) & $1.1(0.2-3.4)$ & $0.9(0.03-6.50)$ & -0.7 & 0.46 \\
\hline
\end{tabular}




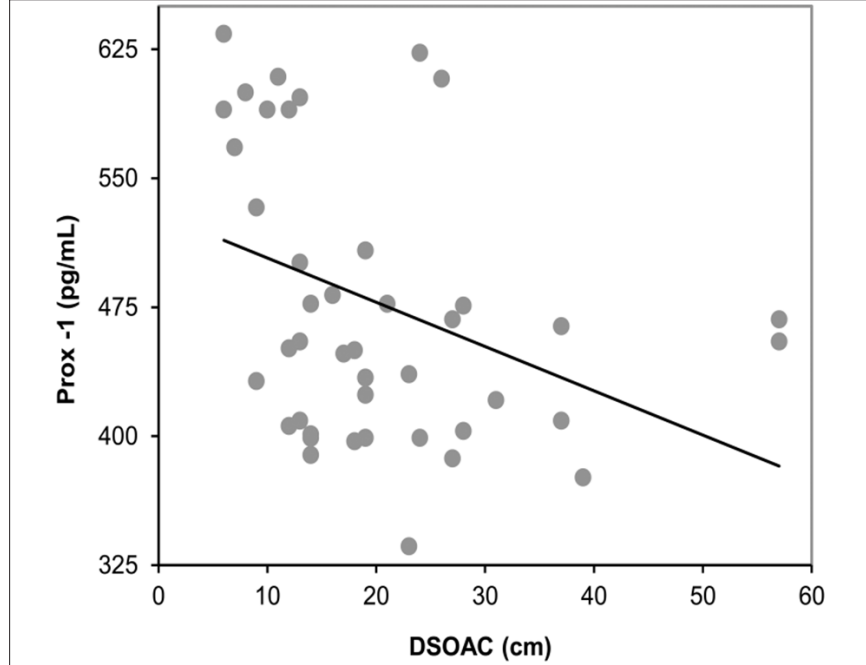

Figure 1. Correlation graph of the relationship between Prox-1 and DSOAC. There was a weak negative correlation between Prox1(Prospero-related homeobox gene-1) levels and DSOAC (Differences between the sums of arm circumferences); $r=-0.417, p<0.01$



Figure 2. Correlation graph between Prox-1 and the number of lymph nodes removed. There was a weak negative correlation between Prox-1(Prospero-related homeobox gene-1) levels and NLNR (number of lymph nodes removed); $r=-0.426, p<0.01$

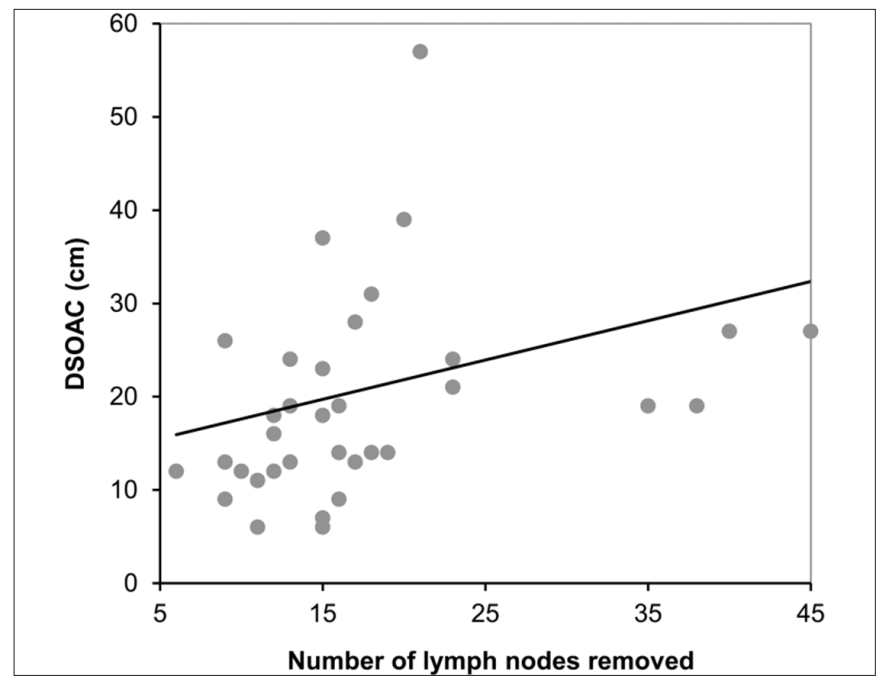

Figure 3. Correlation graph between the DSOAC and the number of lymh nodes removed. There was a positive and moderate correlation between DSOAC (Differences between the sums of arm circumferences) and NLNR (Number of lymph nodes removed); $r=0.533, p<0.001$
Figure 4 shows the results of a ROC curve analysis of the LE and control groups to detect the Prox-1, LVYE1 , and PDPN cutoff values in order to predict patient LE. The results are as follows: the ROC curve for Prox1: $\mathrm{AUC}=0.752$ (95\% $\mathrm{CI}=0.699-0.808)$; sensitivity $=86 \%$, specificity $=66 \%$, cut off value $=477.6 \mathrm{pg} / \mathrm{mL}, \mathrm{p}<0.001$. For LVYE-1: AUC $=0.650 \quad(95 \% \quad \mathrm{CI}=0.589-0.711)$, sensitivity $=70 \%$, specificity $=52 \%$, cut off value $=5.5 \mathrm{ng} /$ $\mathrm{mL}, \mathrm{p}<0.001$. For PDPN: AUC $=0.632$ (95\% CI $=0.567-$ 0.695), sensitivity $=68 \%$, specificity $=54 \%$, cut off value $=1.1 \mathrm{ng} / \mathrm{mL}, \mathrm{p}<0.001$ (Table 4). According to ROC data, Prox-1 is the most useful of these three markers for predicting the presence of LE.

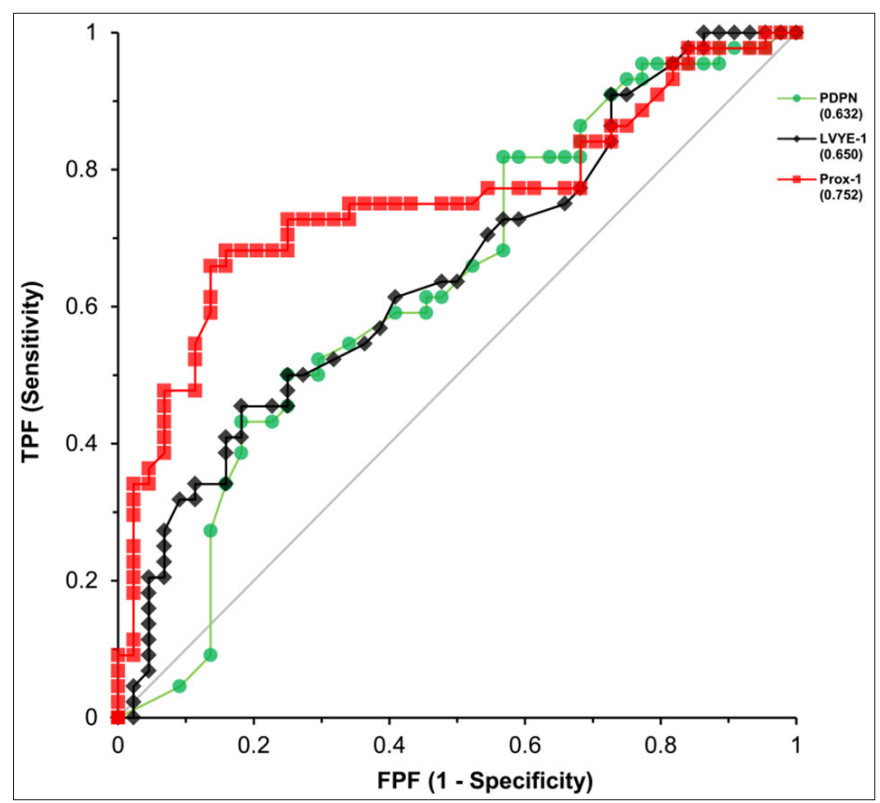

Figure 4. Receiver Operating Characteristic (ROC) Curve of Prox-1, LVYE-1, and PDPN. The ROC curve analysis of the Lymphedema (LE) and control groups to detect the Prox1(Prospero-related homeobox gene-1), LVYE-1 (Lymphatic vessel endothelial receptor-1), and PDPN (Podoplanin) cutoff values. The ROC curve for Prox-1: AUC (Area Under Curve) $=0.752$ (95\% CI (Confidence Interval) $=0.699-0.808)$; sensitivity $=86 \%$, specificity $=66 \%$, cut off value $=477.6 \mathrm{pg} / \mathrm{mL}, \mathrm{p}<0.001$. For LVYE-1: AUC $=0.650(95 \% \mathrm{CI}=0.589-0.711)$, sensitivity $=70 \%$, specificity $=52 \%$, cut off value $=5.5 \mathrm{ng} / \mathrm{mL}, \mathrm{p}<0.001$. For PDPN: AUC $=0.632(95 \% \mathrm{CI}=0.567-0.695)$, sensitivity $=68 \%$, specificity $=54 \%$, cut off value $=1.1 \mathrm{ng} / \mathrm{mL}, \mathrm{p}<0.001$. TPF: True Positive Fractions, FPF:False Positive Fractions

\section{Table 4. Diagnostic properties of markers}

\begin{tabular}{|c|c|c|c|c|c|}
\hline Variables & $\begin{array}{c}\text { AUC } \\
(95 \% \mathrm{CI})\end{array}$ & $\begin{array}{c}\text { Sensitivity } \\
(\%)\end{array}$ & $\begin{array}{c}\text { Specificity } \\
(\%)\end{array}$ & $\begin{array}{c}\text { Cut } \\
\text { off } \\
\text { value }\end{array}$ & $\mathbf{p}$ \\
\hline $\begin{array}{l}\text { Prox-1 } \\
(\mathrm{pg} / \mathrm{mL})\end{array}$ & $\begin{array}{c}0.752 \\
(0.699-0.808)\end{array}$ & 86 & 66 & 477.6 & $<0.001$ \\
\hline $\begin{array}{l}\text { LVYE-1 } \\
\text { (ng/mL) }\end{array}$ & $\begin{array}{c}0.650 \\
(0.589-0.711)\end{array}$ & 70 & 52 & 5 & $<0.001$ \\
\hline $\begin{array}{l}\text { PDPN } \\
\text { (ng/mL) }\end{array}$ & $\begin{array}{c}0.632 \\
(0.567-0.695)\end{array}$ & 68 & 54 & 1.1 & $<0.001$ \\
\hline
\end{tabular}




\section{DISCUSSION}

Over the last 10 years, LEC-specific markers such as 5'-nucleotidase, VEGFR-3, PDPN, Prox-1, and LVYE1 have been introduced to elucidate the molecular mechanisms of LEC, and lymphangiogenesis in particular. LEC markers facilitate the detailed analysis of lymphatic vessel structure, and the structural organization and lymphangiogenesis under physiological and pathological conditions (16). A better understanding of LEC response and behavior under physiological and pathological conditions will allow the development of new treatments for resistant diseases such as malignant tumors, metastasis, and LE.

There are studies describing the risk factors for LE and treatment modality (surgery, radiotherapy) $(5-7,19)$ and the genetic relationships $(20-25,26)$ which predict LE development after breast cancer treatment. The present study found that LEC-specific markers Prox-1, PDPN, and LVYE-1 levels were lower in patients with LE after breast cancer treatment compared to the control group. Therefore, these markers may be predictors of LE development after breast cancer treatment.

LE, especially in advanced stages, can typically be diagnosed following clinical presentation and history, and the patient can subsequently be directed to treatment. The identification of LE is typically performed by circumferential measurements or volumetric documentation that compares the affected arm of the patient to their unaffected arm. Bioimpedance techniques are widely used during physical examination of body composition analysis to provide a more direct measurement of differences in the volume of edema, and, therefore, are a reliable and reproducible method of assessing LE.

However, noticing and distinguishing LE in the early stages presents greater difficulties (1). This is because, in the early phase of LE, pathological changes that occur because of the effects of lymphatic transport dysfunction on lymphatic endothelial cell (LEC) behavior and molecular mechanisms alter the LEC structure, function, and release of LEC-specific markers. In the present study, Prox-1 levels in Stage 2 LE patients were significantly lower than those in Stage 1 patients. Prox-1 also showed a significant negative correlation with the number of lymph nodes removed and DSOAC. In addition, when compared to other LEC-specific markers, such as LVYE1 and PDPN, Prox-1 had the largest area under the ROC curve. This result suggests that Prox-1, which should be at a constant level for the protection of LEC identity, is the earliest affected pathological change occurring with LE. We believe this finding indicates that Prox-1 may therefore be a useful marker in the early detection of LE.
Pan Y et al. (18) demonstrated that PDPN expression was transcriptionally regulated by Prox-1 in cultured murine LECs. In support of this finding, the decrease in serum PDPN levels in the present study may be explained by the decrease in serum Prox-1 levels. Some studies have shown that there is a significant relationship between LE severity and axillary lymph nodes removed, which is frequently applied in breast cancer treatment $(19,27,28)$. We found that Prox-1 levels, thought to be the most specific lineage markers of lymphatic endothelium, were significantly lower in Stage 2 patients than in Stage 1, and have a negative correlation with the number of lymph nodes removed during breast cancer treatment.

Investigation of LEC biological characteristics and mechanisms in the edematous microenvironment may allow the explanation of developmental duration and severity differences of LE in each individual, and also why LE develops only in some people with the same risk factors after cancer treatment. In other words, lymphatic damage alone is not sufficient for the development of LE. The fluid accumulated in LE significantly affects the cellular behavior in the affected area, and stimulates pathological changes such as immune cell infiltration, inflammatory cascade activation, adipose accumulation, and tissue fibrosis.

The Th2 inflammatory response has been suggested to play a key role in the pathogenesis of LE (29). Shin K et al. (30) showed that Th2 cytokines downregulate Prox1 and LVYE-1 LEC markers, and that the blockade of these cytokines smoothed the formation and function of lymphatic vessels in an in vitro asthma model. There is no doubt that LECs are flexible and can adapt their structure to increased lumen pressure and shear stress (12). To observe this condition in vitro, Wang $S$ et al. (15) applied $0 \%$ (control), $4 \%$, and $8 \%$ mechanical stress to purified human LECs, and after 72 hours observed that excessive stretching at an $8 \%$ strain significantly increased LEC proliferation, Prox-1 expression, and lymphangiogenesis. In this case, the first responses of the LECs to stress are proliferation and increased expression of LEC-specific markers, especially Prox-1, and lymphangiogenesis. Even lymphangiogenesis may prevent LE development by increasing lymphatic drainage in the first stage of LE. However, due to increased fibrosis and inflammation in advanced LE, the expression of lymphangiogenesis and LEC-specific markers may be reduced, as shown in the present study.

In this study, average BMI of LE group was significantly higher than that of the control group. The study showed that obesity is a risk factor for secondary developed LE in breast cancer treatment $(1,3,11)$. In a study conducted by the researchers, it was found out that calorie restriction in obese women reduced the levels of TNF- $\alpha$, IL- 6 and 
adhesion molecules, weight loss resulting from calorie restriction regulated the $\mathrm{NO}$ levels and thus, remedied the endothelium cell functions (31). In an another study in which the lymphatic cell changes were analyzed, it was found out that lymphatic density and pumping frequency significantly reduced, lymphatic vessel leakiness increased and the gene expression patterns of LECs changed in obese rats. Furthermore; it was argued that significance of lymphatic specific indicators reduced in obese rats in comparison to the non-fat rats and the aerobic exercises of obese rats both reduced the perilymphatic accumulation of inflammatory cells and remedied specific gene expressions of lymphatic endothelium cells such as VEGFR-3 and Prox-1 (32). The studies to be conducted on obesity and the interaction of LEC markers during the LE development process will have positive effects on protection from LE and even on the treatment process.

\section{Study Limitations}

The sample size, and the absence of a group of patients who had been treated for breast cancer but had not developed LE, are two of the limitations of the present study. Furthermore, the patients in the LE group could not be diagnosed with bioimpedance spectroscopy. Subsequent studies with larger samples and LEC-specific markers in patients with breast cancer therapy who have not developed LE may facilitate a better explanation of the pathophysiological role of LECs in the development of LE

\section{CONCLUSION}

The LEC-specific markers Prox-1, LVYE-1, and PDPN were found to be low in the sera of patients who developed LE following breast cancer treatment. In addition, Prox-1 levels are negatively correlated with the severity of LE. Further research should provide a better understanding of the lymphatic system and LEC functions, allowing the development of new perspectives on LE diagnosis, prognosis, and treatment.

\section{ETHICAL DECLARATIONS}

Ethics Committee Approval: The study approved by the Gaziosmanpaşa Taksim Training and Research Hospital Clinical Researchs Ethics Committee (Date: 2018, Decision No: 81).

Informed Consent: Written informed consent was obtained from all participants who participated in this study.

Referee Evaluation Process: Externally peer-reviewed.

Conflict of Interest Statement: The authors have no conflicts of interest to declare.
Financial Disclosure: The authors declared that this study has received no financial support.

Author Contributions: All of the authors declare that they have all participated in the design, execution, and analysis of the paper, and that they have approved the final version.

Note: Presented at the National Associations of Clinical Biochemistry Professionals annual meeting, Kemer, Antalya, Turkey, October 2018.

\section{REFERENCES}

1. Warren AG, Brorson H, Borud LJ, Slavin SA. Lymphedema: a comprehensive review. Ann Plast Surg 2007; 59: 464-72.

2. Dayan JH, Ly CL, Kataru RP, Mehrara BJ. Lymphedema: pathogenesis and novel therapies. Annu Rev Med 2018; 69: 263 76.

3. Grada AA, Phillips TJ. Lymphedema pathophysiology and clinical manifestations. J Am Acad Dermatol 2017; 77: 1009-20.

4. Cormier JN, Askew RL, Mungovan KS, Xing Y, Ross MI, Armer JM. Lymphedema beyond breast cancer: a systematic review and meta-analysis of cancer-related secondary lymphedema. Cancer 2010; 116: 5138-49.

5. Schunemann $H$, Willich $N$. Lymphedema after breast carcinoma: a study of 5868 cases. Dtsch Med Wochenschr 1997; 122: 536-41.

6. Petrek JA, Senie RT, Peters M, Rosen PP. Lymphedema in a cohort of breast carcinoma survivors 20 years after diagnosis. Cancer 2001; 92: 1368-77.

7. Hinrichs CS, Watroba NL, Rezaishiraz H, et al. Lymphedema secondary to postmastectomy radiation: incidence and risk factors. Ann Surg Oncol 2004; 11: 573-80.

8. Lasinski BB. Complete decongestive therapy for treatment of lymphedema. Semin Oncol Nurs 2013; 29: 20-7.

9. Gerli R, Solito R, Weber E, Aglianó M. Specific adhesion molecules bind anchoring filaments and endothelial cells in human skin initial lymphatics. Lymphology 2000; 33: 148-57.

10. Jurisic G, Detmar M. Lymphatic endothelium in health and disease. Cell Tissue Res 2009; 335: 97-108.

11. Adamczyk LA, Gordon K, Kholová I, et al. Lymph vessels: the forgotten second circulation in health and disease. Virchows Arch 2016; 469: 3-17.

12. Breslin JW, Kurtz KM. Lymphatic endothelial cells adapt their barrier function in response to changes in shear stress. Lymphat Res Biol 2009; 7: 22-37.

13. Miteva DO, Rutkowski JM, Dixon JB, Kilarski W, Shields JD, Schwartz MA. Transmural flow modulates cell and fluid transport functions of lymphatic endothelium. Circ Res 2010; 106: 920-31.

14.Ji RC. Lymphatic endothelial cells, lymphedematous lymphangiogenesis, and molecular control of edema formation. Lymphat Res Biol 2008; 6: 123-37.

15. Wang S, Nie D, Rubin JP, Kokai L. Lymphatic endothelial cells under mechanical stress: altered expression of inflammatory cytokines and fibrosis. Lymphat Res Biol 2017; 15: 130-5.

16. Kato S, Shimoda H, Ji RC, Miura M. Lymphangiogenesis and expression of specific molecules as lymphatic endothelial cell markers. Anat Sci Int 2006; 81: 71-83.

17.Liersch R, Biermann C, Mesters RM, Berdel WE. Lymphangiogenesis in cancer: current perspectives. Recent Results Cancer Res 2010; 180: 115-35.

18. Pan Y, Wang WD, Yago T. Transcriptional regulation of podoplanin expression by Prox 1 in lymphatic endothelial cells. Microvasc Res 2014; 94: 96-102. 
19. Coriddi M, Khansa I, Stephens J, Miller M, Boehmler J, Tiwari P. Analysis of factors contributing to severity of breast cancerrelated lymphedema. Ann Plast Surg 2015; 74: 22-5.

20.Leung G, Baggott C, West C, et al. Cytokine candidate genes predict the development of secondary lymphedema following breast cancer surgery. Lymphat Res Biol 2014; 12: 10-22.

21. Hayes S, Cornish B, Newman B. Comparison of methods to diagnose lymphoedema among breast cancer survivors: 6-month follow-up. Breast Cancer Res Treat 2005; 89: 221-26.

22. Choi YH, Seo KS. Correlation among bioimpedance analysis, sonographic and circumferential measurement in assessment of breast cancer-related arm lymphedema. Lymphology 2014; 47: 123-33.

23. McLaughlin SA, Wright MJ, Morris KT, et al. Prevalence of lymphedema in women with breast cancer 5 years after sentinel lymph node biopsy or axillary dissection: Patient perceptions and precautionary behaviors. J Clin Oncol 2008; 26: 5213-19.

24. Bernas MJ, Witte CL, Witte MH. The diagnosis and treatment of peripheral lymphedema: draft revision of the 1995 Consensus Document of the International Society of Lymphology Executive Committee for discussion at the September 3-7, 2001, XVIII International Congress of Lymphology in Genoa, Italy. Lymphology 2001; 34: 84-91.

25. Miaskowski C, Dodd M, Paul SM, et al. Lymphatic and angiogenic candidate genes predict the development of secondary lymphedema following breast cancer surgery. PLoS One 2013; 8: e60164.

26. Hadizadeh M, Mohaddes Ardebili SM, Salehi M, et al. GJA4/ Connexin 37 Mutations correlate with secondary lymphedema following surgery in breast cancer patients. Biomedicines 2018; 6: 23 .

27.Larson D, Weinstein M, Goldberg I, et al. Edema of the arm as a function of the extent of axillary surgery in patients with stage III carcinoma of the breast treated with primary radiotherapy. Int J Radiat Oncol Biol Phys 1986; 12: 1575-82.

28. Silberman H. Axillary lymphadenectomy for breast cancer: impact on survival. In: Silberman H, Silberman AW (ed) Surgical oncology: multidisciplinary approach to difficult problems. London: Arnold, 2002, pp 369-85.

29. Ly CL, Kataru RP, Mehrara BJ. Inflammatory manifestations of lymphedema. Int J Mol Sci 2017; 18: 171.

30. Shin K, Kataru RP, Park HJ, et al. Th2 cells and their cytokines regulate formation and function of lymphatic vessels. Nat Commun 2015; 6: 6196.

31. Korybalska K, Luczak J, Swora-Cwynar E, et al. Weight lossdependent and-independent effects of moderate calorie restriction on endothelial cell markers in obesity. J Physiol Pharmacol 2017; 68: 597-608

32. Hespe GE, Kataru RP, Savetsky IL, et al. Exercise training improves obesity-related lymphatic dysfunction. J Physiol 2016; 594: 4267-82. 